OPEN ACCESS

Edited by:

Michael Kemp,

University of Southern

Denmark, Denmark

Reviewed by:

Marcia Garnica,

Federal University of Rio de Janeiro, Brazi

Oana Sandulescu,

Carol Davila University of Medicine and Pharmacy, Romania

*Correspondence:

Hai-nan Zhang

hainanzhang@csu.edu.cn

Xiao-mei Wu

wuxiaomei0923@csu.edu.cn

†These authors have contributed equally to this work

Specialty section

This article was submitted to Infectious Diseases - Surveillance,

Prevention and Treatment a section of the journal Frontiers in Medicine

Received: 26 May 2021

Accepted: 11 August 2021 Published: 10 September 2021

Citation:

Zeng S, Yan W-q, Wu X-m and Zhang H-n (2021) Case Report: Diagnosis of Klebsiella pneumoniae

Invasive Liver Abscess Syndrome With Purulent Meningitis in a Patient

From Pathogen to Lesions.

Front. Med. 8:714916.

doi: 10.3389/fmed.2021.714916

\section{Case Report: Diagnosis of Klebsiella pneumoniae Invasive Liver Abscess Syndrome With Purulent Meningitis in a Patient From Pathogen to Lesions}

\author{
Sheng Zeng ${ }^{1}$, Wei-qian $\mathrm{Yan}^{2}$, Xiao-mei $\mathrm{Wu}^{2 * t}$ and Hai-nan Zhang ${ }^{2 * t}$ \\ ${ }^{1}$ Department of Geriatrics, The Second Xiangya Hospital, Central South University, Changsha, China, ${ }^{2}$ Department of \\ Neurology, The Second Xiangya Hospital, Central South University, Changsha, China
}

As a determinant human pathogen, Klebsiella pneumoniae is known to cause rare K. pneumoniae liver abscess syndrome (KLAS) which was more common in Asia in early-stage and reported increasingly outside Asia now. Patients with KLAS who have septic metastatic ocular or central nervous system (CNS) lesions are associated with high morbidity and mortality. Relatively infrequent adult community-acquired $K$. pneumoniae meningitis have been documented and most were with poor prognosis. In this paper, we reported a case of KLAS presenting purulent meningitis as disease onset. While negative results were obtained in the bacterial culture of CSF, blood, or liver pus, metagenomic next-generation sequencing (mNGS) of CSF, and blood samples which were synchronously performed demonstrated Klebsiella pneumoniae as the pathogenic microorganism (13,470 and 5,318 unique reads, respectively). The ultimately cured patient benefited from rapid pathogen diagnosis, early percutaneous drainage of the abscess, and prompt appropriate antibiotic administration. Our case highlights the importance of clinicians using mNGS for early pathogen diagnosis of this disease.

Keywords: $K$. pneumoniae liver abscess syndrome, purulent meningitis, rapid pathogen diagnosis, metagenomic next-generation sequencing (mNGS), case report

\section{INTRODUCTION}

K. pneumoniae is a well-known human nosocomial pathogen although it appears as the normal flora of the human oral cavity and intestine in most situations (1). Most Klebsiella pneumoniae community-acquired infections result in pneumonia or urinary tract infections. However, it also causes rare K. pneumoniae liver abscess (KLA) in the absence of predisposing factors of hepatobiliary disease (2). KLA was reported most in Asia in early-stage and now it is increasing outside Asia $(3,4)$. Except for liver abscess, bacteremia dissemination may result in severe complications including endophthalmitis, meningitis, necrotizing fasciitis, and other illnesses. About $13 \%$ of patients with KLA have septic metastatic ocular or central nervous system (CNS) lesions which are associated with high morbidity and mortality (5). The rapid diagnosis followed by appropriate treatment may improve the patient's outcome.

Metagenomic next-generation sequencing (mNGS) is becoming a powerful pathogen detection tool with the advantages of high sensitivity, wide-coverage, and high efficiency, which can greatly improve pathogen identification (6). It has now been successfully used for pathogen detection in biological specimens including CSF and blood (7). Early diagnosis assisted by mNGS contributes 
to timely treatment. In our report, we displayed a case of KLA manifested as purulent meningitis at the onset of the disease. Rapid pathogen diagnosis facilitated further lesion detection, proper treatment, and full recovery of the patient. Until now, few KLA cases with metastatic meningitis were reported, fewer patients achieved a good prognosis (8). Our case suggested the importance of early pathogen diagnosis.

\section{CASE PRESENTATION}

A 59-year-old female rural resident in Hunan province, China, was admitted to the Emergency Department (ED) of Second Xiangya Hospital, Central South University. She complained of headache with fever for 6 days and fatigue and somnolence for 1 day. The first symptoms were headache with fever. She experienced four episodes of diarrhea on the first day. No other gastrointestinal symptoms were presented since then. From the second day to the fourth day, she was admitted to a local hospital. A lumbar puncture was performed because of clinical suspicion of "intracranial infection." The intracranial pressure was 140 $\mathrm{mmH}_{2} \mathrm{O}$. Cerebrospinal fluid (CSF) was yellow and purulent, and revealed 1,080 white blood cells/ $\mu \mathrm{L}$ with a monocyte's ratio of $56.9 \%$. She was immediately treated with intravenous ceftriaxone $2,000 \mathrm{mg} \mathrm{q} 12 \mathrm{~h}$. Although the history of diabetes was denied, her random blood glucose was as high as $14 \mathrm{mmol} / \mathrm{L}$ during hospitalization. On the fifth day, she was recommended to refer to our hospital due to the new appearance of fatigue and somnolence. Her past medical history showed no recent travel, tick bites, sick contact, alcohol, or drug use. Upon admission, her initial vital signs included a body temperature of $36.7^{\circ} \mathrm{C}$, heart rate of 82 beats/min, blood pressure of 125/80 $\mathrm{mmHg}$, respiratory rate of 23 breaths/min, and oxygen saturation of $99 \%$ on $2 \mathrm{~L} / \mathrm{min}$ oxygen. Her consciousness was somnolence. No skin rash was observed, and no obvious abnormality was found on physical examination of the heart, lung, and abdomen. A neurologic examination revealed that the neck was stiff and Kernig's sign was positive. Laboratory test on ED showed a white blood cell count of $9.0 \times 10^{9} / \mathrm{L}$ with an elevated neutrophil ratio of $83.9 \%$ in blood routine test. Liver function test showed hypoalbuminemia $(26.1 \mathrm{~g} / \mathrm{L})$. There was no significant change in the coagulation panel. The random blood glucose was 15.3 $\mathrm{mmol} / \mathrm{L}$. The blood ketone and lactate were 2.43 and $0.9 \mathrm{mmol} / \mathrm{L}$, respectively. Lung computed tomography (CT) scan showed focal small patchy infiltrates in both lower lungs. Considering the severity of the patient's condition, she was sent to the intensive care unit of the department of neurology (NICU) for further treatment. Further laboratory tests and auxiliary examinations were arranged. The concentrations of procalcitonin, C-reactive protein, erythrocyte sedimentation rate (ESR) and interleukin 6 (IL-6) were $8.720 \mathrm{ng} / \mathrm{ml}, 134.0 \mathrm{mg} / \mathrm{L}, 45 \mathrm{~mm} / \mathrm{h}$, and $630.0 \mathrm{pg} / \mathrm{ml}$, respectively. The result of glycosylated hemoglobin was $10.7 \%$. Thyroid function test results showed that T3 was $0.56 \mathrm{~mol} / \mathrm{L}, \mathrm{FT} 3$ was $1.58 \mathrm{~mol} / \mathrm{L}$, and T4 was $50.70 \mathrm{~mol} / \mathrm{L}$. Two sets of peripheral blood cultures were ordered. Lumbar puncture showed the intracranial pressure was $130 \mathrm{mmH}_{2} \mathrm{O}$. Cerebrospinal fluid (CSF) revealed 1,000 white blood cells/ $\mu \mathrm{L}$ with proportion of multinucleated cells of $95 \%$, protein of $943 \mathrm{mg} / \mathrm{L}$, and glucose of $3.97 \mathrm{mmol} / \mathrm{L}$ (synchronous blood glucose of $14.0 \mathrm{mmol} / \mathrm{L}$ ). The CSF Gram staining, Ink staining, acid-fast staining, and bacterial culture were all negative. The above findings led to the diagnosis of purulent meningitis, pulmonary infection, diabetes mellitus (DM), and diabetic ketosis. To further identify the pathogen, the PACEseq mNGS test (Hugobiotech, Beijing, China) was also performed on CSF samples on the Nextseq 550 platform (Illumina, San Diego, CA). She has immediately received emergency management of ketosis and was treated with intravenous meropenem 2,000 $\mathrm{mg} \mathrm{q} 8 \mathrm{~h}$ and vancomycin $500 \mathrm{mg}$ q6h on admission (at this moment, the duration of ceftriaxone therapy was 3 days).

On the second day of hospitalization, a brain MRI scan was performed, and partial enhancement of the pia mater was recommended (Figures 1A,B). She experienced a chill with a body temperature of $38.6^{\circ} \mathrm{C}$ on this day. Peripheral blood culture was performed. Lumbar puncture and intrathecal injection of $20 \mathrm{mg}$ vancomycin were arranged continuously on the second to the fourth day.

On the fourth day of hospitalization, the mNGS of CSF reported Klebsiella pneumoniae, with a total of 13,470 detected unique reads detected (Figure 2A). Klebsiella pneumoniae invasive liver abscess syndrome was suspected as the clinical features and unusual findings of the pathogen in this patient. An abdominal enhanced CT demonstrated a single abscess $(67 \mathrm{~mm} \times 62 \mathrm{~mm})$ in the right lobe of the liver with separating enhancement (Figures 1C,D) and confirmed our suspicion. No other intra-abdominal pathologies, such as gallstones, were observed. To quickly ascertain the transmission route, mNGS of a blood sample was also arranged. After the diagnosis with Klebsiella pneumoniae invasive liver abscess syndrome, the antimicrobials of the patient were adjusted to meropenem 2,000 mg q8h intravenously. An emergency CTguided percutaneous drainage of the liver abscess was performed, which drained $210 \mathrm{~mL}$ of yellow pus over the first $24 \mathrm{~h}$. The liver aspirate was subjected to Gram staining and both aerobic and anaerobic culture. Repeated blood and liver pus cultures were required during episodes of fever.

On the seventh day of hospitalization (the third day after operation), only $20 \mathrm{~mL}$ yellow pus was drained over the third $24 \mathrm{~h}$. Reexamination of abdominal CT suggested the liver abscess was reduced to $51 \mathrm{~mm} \times 37 \mathrm{~mm}$ (Figure 1E). Lumbar puncture after operation showed the intracranial pressure was $140 \mathrm{mmH}_{2} \mathrm{O}$. Cerebrospinal fluid (CSF) revealed 1 white blood cells $/ \mu \mathrm{L}$ (mononuclear cells), protein $780 \mathrm{mg} / \mathrm{L}$, and glucose 5.88 $\mathrm{mmol} / \mathrm{L}$ (synchronous blood glucose of $8.1 \mathrm{mmol} / \mathrm{L}$ ). Meanwhile, the blood mNGS results demonstrated 5,318 unique reads of Klebsiella pneumoniae (Figure 2B). Thus, purulent meningitis with Klebsiella pneumoniae was septic metastatic from invasive liver abscess through blood.

On the ninth day of hospitalization (the fourth day after operation), the patient recovered to good condition after treatment. However, the patients and their families strongly requested to return to the local hospital for further treatment. A telephone follow-up after 3 months showed the patient recovered and was discharged from the hospital after 29 days of treatment. 

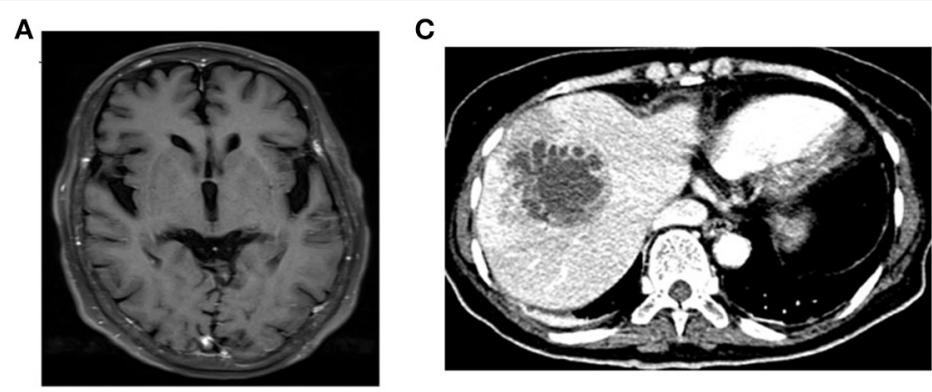

\section{E}
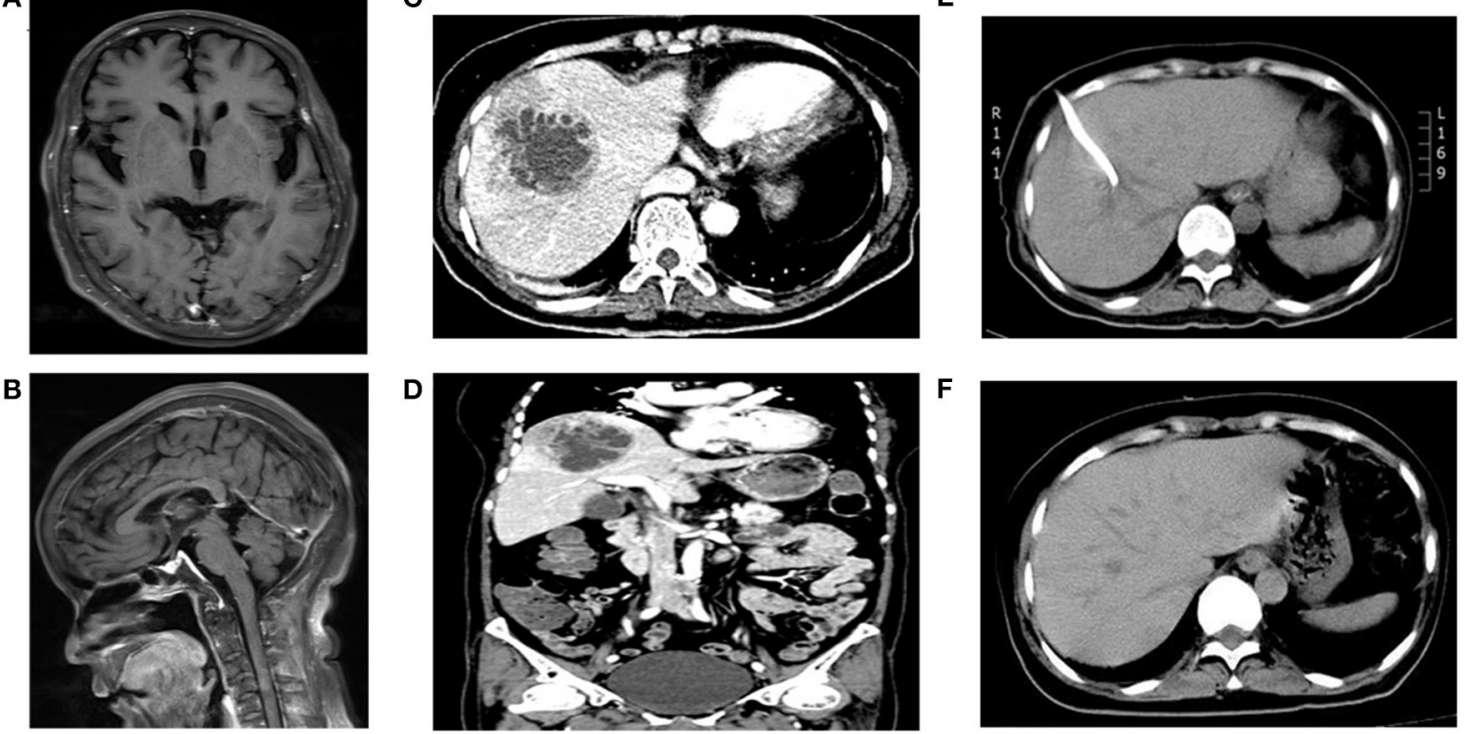

FIGURE 1 | MRI or CT scan images of the patient. (A,B) indicated partial enhancement of pia mater in brain enhanced MRI; (C,D) revealed an area of abnormal attenuation measuring $67 \mathrm{~mm} \times 62 \mathrm{~mm}$ in the right lobe of the liver, indicative of a single large multi-loculated abscess in abdominal enhanced $\mathrm{CT}$; (E) displayed the abscess reduced to $51 \mathrm{~mm} \times 37 \mathrm{~mm} 3$ days after emergency CT-guided percutaneous drainage of the liver abscess in abdominal plain CT; (F) showed no lesions in liver in the reexamination of abdominal CT after full recovery.
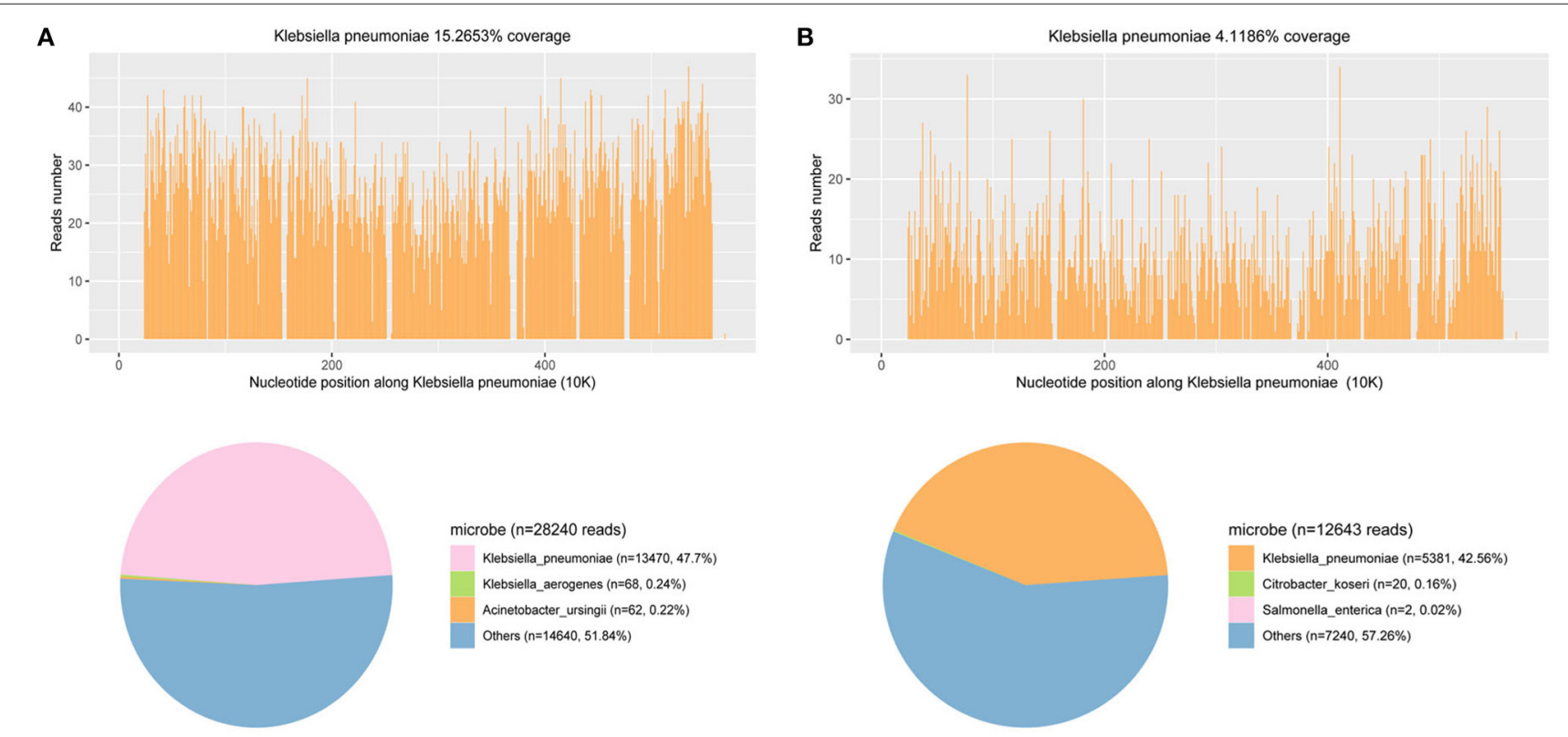

FIGURE 2 | mNGS results of pathogen identification: 47.7 and $42.56 \%$ of bacterial reads corresponded to $K$. pneumoniae with coverage of 15.2653 and $4.1186 \%$ in CSF (A) and blood (B), respectively.

Thus, the total duration of treatment was nearly 40 days. The patient underwent abdominal CT again, which showed no lesions in her liver (Figure 1F) at the second follow-up after 9 months.

It should be noted that bacterial cultures of CSF, blood, and liver pus in the whole disease course were all negative.

\section{DISCUSSION}

Klebsiella pneumoniae liver abscess syndrome (KLAS) is clinically characterized by bacteremia, liver abscesses, and metastatic infection caused by hypervirulent strains harboring capsular 
serotype $\mathrm{K} 1$ or $\mathrm{K} 2$. DM may be a risk factor for this syndrome (2). The most common clinical manifestations of patients with $K$ pneumoniae liver abscesses are fever, chills, and abdominal pain. However, all these manifestations lack specificity. For patients, especially those who suffer from diabetes mellitus present with $K$ pneumoniae bacteraemia, meningitis, endophthalmitis, or other extrahepatic infections, it is necessary to look for potential liver abscess. Thus, timely identification of pathogens is the key to better management. However, traditional methods for pathogen discovery such as bacterial culture are time-consuming and lack sensitivity (9). The positivity rate of traditional methods is influenced by quality and quantity of specimens, patient antibiotic administration, the severity of infection, and laboratory sufficiency. In our case, the effort was paid through the repeated bacterial cultures of CSF, blood, or liver pus. However, the results were poor and were most likely influenced by empirical antibiotic therapy. The patient's treatment could be delayed without the identification of the pathogen by mNGS.

mNGS emerges as an alternative and efficient molecular diagnostic method, which overcomes the limitations of traditional methods. It is now increasingly applied in the identification of pathogens in clinical practice, such as sepsis, meningitis, and acute respiratory infection $(7,10,11)$. The stateof-the-art mNGS technology has advantages in identifying rare, novel, difficult-to-detect, and coinfected pathogens directly from clinical samples, providing timely diagnostic evidence to guide treatment plans (12). In the future, more validated workflows, lower cost, and simplified interpretation criteria would be needed to further routinely implement mNGS in clinical practice. Our patient was an elderly rural woman who had an unrecognized history of DM. She presented with purulent meningitis at the time of onset and was demonstrated as KLAS on the fourth day after admission. After consulting the literature, we found that adult community-acquired $K$. pneumoniae meningitis has been less recorded $(8,13)$. Unfortunately, few patients have survived this disease. In addition to the severity of the disease, the lack of timely diagnosis and standardized treatment may also be the important reasons.

Until now, there are no clear guidelines for the management of Klebsiella pneumoniae invasive liver abscess syndrome with purulent meningitis (2). The basic consensus is the combination of early percutaneous drainage or open (laparoscopic) surgical drainage of the abscess and prompts the appropriate antibiotic administration. The selection of antibiotics is based on invitro susceptibilities and clinical response. However, results

\section{REFERENCES}

1. Holt KE, Wertheim H, Zadoks RN, Baker S, Whitehouse CA, Dance D, et al. Genomic analysis of diversity, population structure, virulence, and antimicrobial resistance in Klebsiella pneumoniae, an urgent threat to public health. Proc Natl Acad Sci USA. (2015) 112:E3574-81. doi: 10.1073/pnas.1501049112

2. Siu LK, Yeh KM, Lin JC, Fung CP, Chang FY. Klebsiella pneumoniae liver abscess: a new invasive syndrome. Lancet Infect Dis. (2012) 12:8817. doi: 10.1016/S1473-3099(12)70205-0 of antibiotics susceptivity are time-consuming. Therefore, the empirical use of high-dose third-generation cephalosporins, including cefotaxime (up to $2,000 \mathrm{mg}$ every $4 \mathrm{~h}$ ) and ceftriaxone (2,000 mg, twice a day) are options for treatment of $K$. pneumoniae meningitis. Imipenem and meropenem can be given to patients when strains containing extended-spectrum beta-lactamase are suspected. Our patient benefited from the empirically and sufficiently intravenous use of meropenem and early percutaneous drainage of abscesses in our clinic.

In conclusion, we shared a case of KLA that presented with purulent meningitis at the onset of the disease. Rapid pathogen diagnosis through mNGS facilitated further lesion detection, proper treatment, and full recovery of the patient. Nowadays, adult community-acquired $K$. pneumoniae meningitis carries a very poor prognosis. Our case highlights the importance of early pathogen diagnosis by mNGS for the precise treatment of this disease. Meantime, it raises the potential of diagnosing severe infectious diseases from pathogens to lesions.

\section{DATA AVAILABILITY STATEMENT}

The datasets used and/or analyzed during the current study are available from the corresponding author on reasonable request.

\section{ETHICS STATEMENT}

Ethical Committee of the Second Xiangya Hospital of the Central South University in China (equivalent to an Institutional Review Board) approved the study, and written informed consent was obtained from the patient for publication of this Case Report and any accompanying images.

\section{AUTHOR CONTRIBUTIONS}

SZ collected the materials, analyzed the data, and wrote the paper. $\mathrm{Wq}-\mathrm{Y}$ assisted in clinical follow up. $\mathrm{Xm}-\mathrm{W}$ and $\mathrm{Hn}-\mathrm{Z}$ designed the concept and provided administrative support. All authors contributed to the article and approved the submitted version.

\section{FUNDING}

This work was supported by the Program of National Natural Science Foundation of China (\#81801123).
3. Chang FY CM, Fan RL, Shaio MF. A clinical study of Klebsiella liver abscess. Taiwan Yi Xue Hui Za Zhi. (1988) 87:282-87.

4. Vila A, Cassata A, Pagella H, Amadio C, Yeh KM, Chang FY, et al. Appearance of Klebsiella pneumoniae liver abscess syndrome in Argentina: case report and review of molecular mechanisms of pathogenesis. Open Microbiol J. (2011) 5:107-13. doi: 10.2174/1874285801105010107

5. Fang CT, Lai SY, Yi WC, Hsueh PR, Liu KL, Chang SC. Klebsiella pneumoniae genotype K1: an emerging pathogen that causes septic ocular or central nervous system complications from pyogenic liver abscess. Clin Infect Dis. (2007) 45:284-93. doi: $10.1086 / 519262$ 
6. Miao Q, Ma Y, Wang Q, Pan J, Zhang Y, Jin W, et al. Microbiological diagnostic performance of metagenomic next-generation sequencing when applied to clinical practice. Clin Infect Dis. (2018) 67:S231-40. doi: 10.1093/cid/ciy693

7. Wilson MR, Sample HA, Zorn KC, Arevalo S, Yu G, Neuhaus J, et al. Clinical metagenomic sequencing for diagnosis of meningitis and encephalitis. N Engl J Med. (2019) 380:2327-40. doi: 10.1056/NEJMoa1803396

8. Sun R, Zhang H, Xu Y, Zhu H, Yu X, Xu J. Klebsiella pneumoniae-related invasive liver abscess syndrome complicated by purulent meningitis: a review of the literature and description of three cases. BMC Infect Dis. (2021) 21:15. doi: 10.1186/s12879-020-05702-3

9. Xing XW, Zhang JT, Ma YB, He MW, Yao GE, Wang W, et al. Metagenomic next-generation sequencing for diagnosis of infectious encephalitis and meningitis: a large, prospective case series of 213 patients. Front Cell Infect Microbiol. (2020) 10:88. doi: 10.3389/fcimb.2020.00088

10. Chiu CY, Miller SA. Clinical metagenomics. Nat Rev Genet. (2019) 20:34155. doi: 10.1038/s41576-019-0113-7

11. Chen Y, Feng W, Ye K, Guo L, Xia H, Guan Y, et al. Application of metagenomic next-generation sequencing in the diagnosis of pulmonary infectious pathogens from bronchoalveolar lavage samples. Front Cell Infect Microbiol. (2021) 11:541092. doi: 10.3389/fcimb.2021.541092

12. Gu W, Miller S, Chiu CY. Clinical metagenomic next-generation sequencing for pathogen detection. Annu Rev Pathol. (2019) 14:319-38. doi: 10.1146/annurev-pathmechdis-012418-012751
13. Qian Y, Wong CC, Lai SC, Lin ZH, Zheng WL, Zhao H, et al. Klebsiella pneumoniae invasive liver abscess syndrome with purulent meningitis and septic shock: a case from mainland China. World J Gastroenterol. (2016) 22:2861-6. doi: 10.3748/wjg.v22.i 9.2861

Conflict of Interest: The authors declare that the research was conducted in the absence of any commercial or financial relationships that could be construed as a potential conflict of interest.

Publisher's Note: All claims expressed in this article are solely those of the authors and do not necessarily represent those of their affiliated organizations, or those of the publisher, the editors and the reviewers. Any product that may be evaluated in this article, or claim that may be made by its manufacturer, is not guaranteed or endorsed by the publisher.

Copyright (c) 2021 Zeng, Yan, Wu and Zhang. This is an open-access article distributed under the terms of the Creative Commons Attribution License (CC BY). The use, distribution or reproduction in other forums is permitted, provided the original author(s) and the copyright owner(s) are credited and that the original publication in this journal is cited, in accordance with accepted academic practice. No use, distribution or reproduction is permitted which does not comply with these terms. 